Pacific Journal of Mathematics

RELATIVE FUNCTOR REPRESENTABILITY 


\title{
RELATIVE FUNCTOR REPRESENTABILITY
}

\author{
John L. MacDonald
}

This paper deals with the general problem of determining conditions under which the representability of a given functor $G: \mathbf{A} \rightarrow \mathbf{E} n s$ implies the representability of a subfunctor $F: \mathrm{B} \rightarrow \mathrm{E} n s$ of the restriction of $G$ to a subcategory $\mathbf{B}$ of $\mathbf{A}$. With suitable conditions on $A$ and $B$ a set of necessary and sufficient conditions for the representability of such a functor $F$ can be obtained. A few examples are given which indicate the connection between this case of relative or induced representability and universal algebra.

If $\boldsymbol{A}$ is a suitably restricted category, then a theorem giving a set of necessary and sufficient conditions for a functor $G: \boldsymbol{A} \rightarrow \boldsymbol{E} n s$ to be representable can be proved starting from the same concepts developed for relative representability in the first section. This result on absolute representability is similar to one of Benabou and has as corollary the theorem of Freyd giving a set of conditions for the existence of adjoint functors. We use the convention throughout that the functor $T: \boldsymbol{A} \cdots \boldsymbol{B}$ has an adjoint $S: \boldsymbol{B} \rightarrow \boldsymbol{A}$ or that $T$ is a coadjoint of $S$ if the Hom functors $\boldsymbol{A}(S-,-)$ and $\boldsymbol{B}(-, T-): \boldsymbol{B}^{o p} \times \boldsymbol{A} \cdots \boldsymbol{E} n s$ are naturally isomorphic.

1. Minimal factorizations. Suppose that $G: \boldsymbol{A} \rightarrow \boldsymbol{E} n s$ is a functor and that $\boldsymbol{E} n s$ is the category of sets. Let $\boldsymbol{A}_{\theta}$ be the category whose objects are those pairs $(A, x)$ with $A \in \boldsymbol{A}$ and $x \in G A$ and whose morphisms $\alpha:(A, x) \cdots(B, y)$ are those morphisms $\alpha: A \rightarrow B$ of $A$ such that $(G \alpha) x=y$.

$\boldsymbol{A}$ has minimal $G$ factorizations if for each $(A, x)$ in $\boldsymbol{A}_{\theta}$ there is a subobject $\kappa: K \rightarrow A$ of $A$ in $A$ minimal with respect to the property that $\kappa:(K, k) \rightarrow(A, x)$ for some $k \in G K$. In addition it is required that if $\alpha$ and $\beta$ are morphisms $(K, k) \rightarrow(B, y)$ then $\alpha=\beta$. We call $x=G(\kappa) k$ a minimal $G$ factorization of $x$.

$\boldsymbol{A}$ is well powered if the class of subobjects of each object is a set. The term co-well powered is defined dually. $\boldsymbol{A}$ is complete if each set of objects of $\boldsymbol{A}$ has a product and any pair of morphisms $\alpha, \beta: A \rightarrow B$ has an equalizer.

$G: A \rightarrow E n s$ is continuous if $G$ preserves products and equalizers.

Lemma. If $\boldsymbol{A}$ is a well powered complete category and if $G: \boldsymbol{A} \rightarrow \boldsymbol{E} n s$ is continuous, then $\boldsymbol{A}$ has minimal $G$ factorizations.

Proof. Let $\lambda: E \rightarrow A$ be the intersection of the set $S=$ 
$\left\{\rho_{i}: A_{i} \cdots A\right\}_{i \in I}$ of all subobjects of $A$ such that $\rho_{i}:\left(A_{i}, x_{i}\right) \rightarrow(A, x)$ for some $x_{i} \in G A_{i}$.

Let $P=\Pi_{i \epsilon_{I}}\left(A_{i}\right)$ with projections $\varepsilon_{i}$. For the product $\Pi_{I \times I}(A)$ with projections $p_{i k}$ there exists a unique pair of morphisms $\alpha, \beta: P \cdots \Pi_{I \times I}(A)$ such that
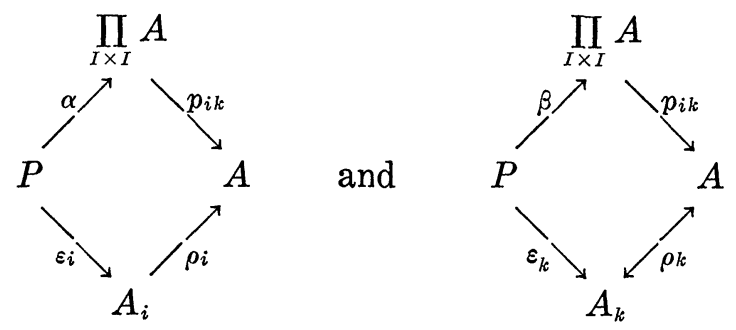

commute for each $i, k \in I$. There exists an equalizer $\kappa$ of $\alpha, \beta$ with $\lambda=\rho_{k} \varepsilon_{k} \kappa: E \rightarrow A$.

For each $\rho_{i} \in S$ let $x_{i} \in G A_{i}$ be an element such that $\rho_{i}:\left(A_{i}, x_{i}\right) \ldots \rightarrow$ $(A, x)$. Since $G$ preserves products there is a unique $\xi \in \Pi_{I} G A_{i}=$ $G\left(\Pi A_{i}\right)$ such that $x_{i}=\left(G \varepsilon_{i}\right) \xi$ for each $i \in I$. Thus $\varepsilon_{i}:(P, \xi) \cdots\left(A_{i}, x_{i}\right)$. From the diagram it follows that $p_{i k} \alpha, p_{i k} \beta:(P, \xi) \cdots(A, x)$ for each $(i, k) \in I \times I$. Thus $(G \alpha) \xi=(G \beta) \xi$ and since $G$ preserves equalizers there is a unique $\varphi$ such that

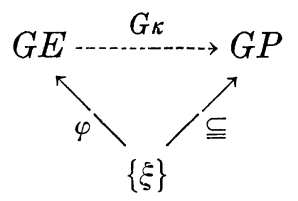

commutes. Thus $\lambda=\rho_{i} \varepsilon_{i} \kappa:(E, \varphi \xi) \rightarrow(A, x)$ as required.

If $\mu, \omega:(E, \varphi \xi) \cdots(B, y)$, then let $\tau: T \rightarrow E$ be the equalizer in $\boldsymbol{A}$ of $\mu, \omega$. Since $G$ preserves equalizers there is a unique $\delta$ such that

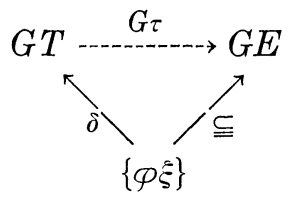

commutes. Thus $\tau:(T, \delta \varphi \xi) \cdots(E, \varphi \xi)$ must be an equivalence since otherwise we would have a contradiction to the minimality of $\lambda: E \rightarrow A$.

A morphism $x: R \rightarrow A$ admits an image if there exists a smallest subobject $\kappa: K \rightarrow A$ such that $x$ has a factorization $R \rightarrow K \cdots A$ with $R \leftrightarrow K$ epic.

COROLlaRY. In a well powered, complete category every morphism $x: R \rightarrow A$ admits an image. 
Proof. The minimal $G=\boldsymbol{A}(R,-)$ factorization of $x$ is the one required.

Corollary. (Freyd) If $\boldsymbol{A}$ is well powered and complete and $J: \boldsymbol{A} \rightarrow \boldsymbol{B}$ is continuous, then for every morphism $y: B \cdots J A$ there is a minimal subobject of $A$ which allows $y$.

Proof. Let $G=\boldsymbol{B}(B, J-)$.

2. Absolute representability. The functor $G: A \rightarrow E n s$ is representable if there exists an object $U$ in $\boldsymbol{A}$ such that the Hom functor $\boldsymbol{A}(U,-)$ is naturally isomorphic to $G$. Equivalently, $G: \boldsymbol{A} \rightarrow \boldsymbol{E} n s$ is representable if $\boldsymbol{A}_{G}$ has an initial point (cf. Mac Lane [7]).

THEOREM. If $\boldsymbol{A}$ is a well powered and complete category, then $G: \boldsymbol{A} \rightarrow \boldsymbol{E} n s$ is representable if and only if

(i) $G$ is continuous.

(ii) There exists a set of objects $\left(A_{i}, a_{i}\right)$ in $A_{\theta}$, indexed by $I$, such that for each $(A, x)$ in $A_{\theta}$ there exists $\left(A_{i}, a_{i}\right) \cdots(A, x)$ for some $i$ in $I$.

Proof. If $\phi: \boldsymbol{A}(R,-) \rightarrow \rightarrow G$ is a natural isomorphism for some $R \in A$, then $\left(R, \phi_{R}\left(1_{R}\right)\right)$ is initial in $\boldsymbol{A}_{G}$ and the continuity of $G$ follows from that of $A(R,-)$.

For the converse we will find an initial point for $\boldsymbol{A}_{G}$. Let $B=$ $\Pi_{j \in I}\left(A_{j}\right)$ with projections $\varepsilon_{j}$. There exists a unique $b \in G B$ with $j$-th component $a_{j}$ since $G$ is continuous. Thus we have $\varepsilon_{j}:(B, b) \cdots\left(A_{j}, a_{j}\right)$ in $\boldsymbol{A}_{G}$. By the Lemma there exists $\kappa:\left(B^{\prime}, b^{\prime}\right) \cdots(B, b)$ giving a minimal $G$ factorization of $b$. If $(A, x) \in \boldsymbol{A}_{\theta}$, then by hypothesis there exists $\varphi:\left(A_{i}, a_{i}\right) \cdots(A, x)$ for some $i \in I$. Thus $\varphi \varepsilon_{i} \kappa:\left(B^{\prime}, b^{\prime}\right) \cdots(A, x)$. If $\beta:\left(B^{\prime}, b^{\prime}\right) \cdots(A, x)$, then $\beta=\phi \varepsilon_{i} \kappa$ by the definition of minimal $G$ factorizations. Hence $\left(B^{\prime}, b^{\prime}\right)$ is the required initial point.

This theorem has the following result of Freyd [4] as a corollary.

Corollary. Let $\boldsymbol{A}$ be a well powered and complete category and let $J: \boldsymbol{A} \cdots \boldsymbol{B}$ be a functor. Then $J$ has an adjoint if and only if

(i) $J$ is continuous

(ii) For $B$ in $\boldsymbol{B}$ there is a set of objects $S_{B} \subseteq \boldsymbol{A}$ such that for $B \rightarrow J A$ with $A \in A$ there is $a_{i}: B \rightarrow J A_{i}$ with $A_{i} \in S_{B}$ and $\alpha: A_{i} \cdots A$ such that 


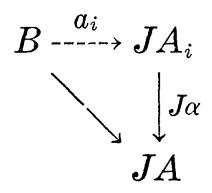

commutes in $\boldsymbol{B}$.

3. Relative representability. Let

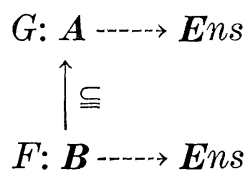

be a diagram of categories and functors such that $F B \cong G B$ for all $B$ in $\boldsymbol{B}$ and $F \beta$ is the restriction of $G \beta$ to $F B$ for $\beta: B \cdots B^{\prime}$ in $\boldsymbol{B}$. Such a functor $F$ will be called a subfunctor of the restriction of $G$ to $\boldsymbol{B}$. Then $\rho \in G B$ is $F$ distinguished if $B \in \boldsymbol{B}$ and $\rho \in F B$.

Now we come to a useful set of conditions sufficient to ensure the representability of a subfunctor $F: \boldsymbol{B} \cdots \boldsymbol{E} n s$ of the restriction of a representable functor $G: \boldsymbol{A} \cdots \boldsymbol{E} n s$ to $\boldsymbol{B} \subseteq \boldsymbol{A}$.

THEOREM. Let $\boldsymbol{A}$ be well and co-well powered and complete, and let $\boldsymbol{B}$ be a full subcategory of $\boldsymbol{A}$ containing a copy of $\Pi B_{i}$ for each set $B_{i}$ of its objects. Suppose that $F$ is a product preserving subfunctor of the restriction of $\boldsymbol{A}(R,-)$ to $\boldsymbol{B}$, with the property that if $\rho$ is $F$ distinguished, than $\rho$ has an image

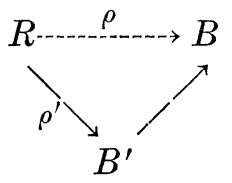

where $\rho^{\prime}$ is $F$ distinguished. Then $F$ is representable by a natural equivalence $\psi: F \rightarrow \boldsymbol{B}\left(R^{\prime},-\right)$ such that the diagram

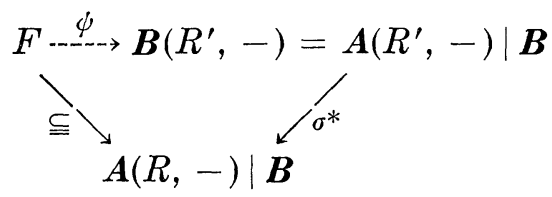

commutes for an epic $\sigma: R \rightarrow R^{\prime}$ in $\boldsymbol{A}$.

Proof. If $\left\{B_{k}\right\}_{k \in K}$ is a set of objects in $\boldsymbol{B}$, then there exists $\Pi_{K}\left(B_{k}\right)$ in $\boldsymbol{B}$ with projections $\varepsilon_{k}: \Pi\left(B_{k}\right) \rightarrow B_{k}$. Then for each $\rho: R \rightarrow \Pi\left(B_{k}\right)$ for which $\varepsilon_{k} \rho: R \rightarrow B_{k}$ is $F$ distinguished for all $k \in K$, 
it follows that $\rho$ is $F$ distinguished since $F$ is product preserving.

Let $S=\left\{X_{j}: R \rightarrow B_{j}\right\}_{j \in J}$ be the set of all $F$ distinguished quotient objects of $R$. There exists a unique $\mu: R \rightarrow \prod_{j \in J} B_{j}$ such that

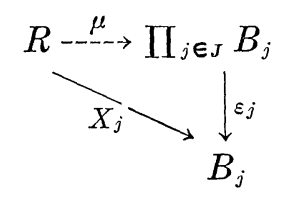

commutes in $\boldsymbol{A}$ for each $j \in J$ where $\varepsilon_{j}$ is the projection. But $X_{j}=$ $\varepsilon_{j} \mu$ is $F$ distinguished. Hence $\mu$ is $F$ distinguished. Let

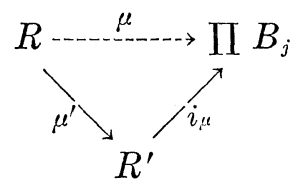

be an image of $\mu$. Then $\mu^{\prime}$ is $F$ distinguished and epic in $\boldsymbol{A}$. Thus $\left(R^{\prime}, \mu^{\prime}\right) \in \boldsymbol{B}_{F}$.

If $(C, \alpha)$ is an object of $\boldsymbol{B}_{F}$, then let

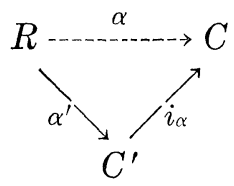

be an image of $\alpha . \quad \alpha^{\prime}$ is epic and is $F$ distinguished since $\alpha$ is $F$ distinguished. Hence $\alpha^{\prime}$ represents a member of $S$. Let $\varepsilon_{\alpha^{\prime}}: \Pi B_{j} \cdots C^{\prime}$ be the corresponding projection. Thus we obtain $i_{\alpha} \varepsilon_{\alpha^{\prime}} i_{\mu}:\left(R^{\prime}, \mu^{\prime}\right) \cdots$ $(C, \alpha)$ in $\boldsymbol{B}_{F}$ from the preceding three diagrams noting that

$$
R \stackrel{\mu}{\rightarrow} \Pi \Pi B_{j} \stackrel{\varepsilon_{\alpha^{\prime}}}{\rightarrow} C^{\prime}=R \stackrel{\alpha^{\prime}}{\rightarrow} C^{\prime} .
$$

But diagram II gives a minimal $\boldsymbol{A}(R,-)$ factorization of $\mu$. Hence if $i_{\alpha} \varepsilon_{\alpha^{\prime}} i_{\mu}$ and $\tau$ are morphisms $\left(R^{\prime}, \mu^{\prime}\right) \cdots(C, \alpha)$ in $\boldsymbol{A}_{F}$ then $i_{\alpha} \varepsilon_{\alpha^{\prime}} i_{\mu}=\tau$. Thus $\left(R^{\prime}, \mu^{\prime}\right)$ is initial in $\boldsymbol{B}_{F}$. If $\psi: F \rightarrow \boldsymbol{B}\left(R^{\prime},-\right)$ is the corresponding equivalence, then for $\sigma=\mu^{\prime}$ it is clear that the required diagram commutes.

The category $\boldsymbol{B} \leqq \boldsymbol{A}$ is closed under subobjects means that if $B \in \boldsymbol{B}$ and $A \rightarrow B$ is a monomorphism in $\boldsymbol{A}$, then $A \in \boldsymbol{B}$.

Finally we obtain a set of necessary and sufficient conditions for relative representability.

Theorem. Let $\boldsymbol{A}$ be well and co-well powered and complete and let $\boldsymbol{B}$ be a full subcategory of $\boldsymbol{A}$ closed under subobjects and containing a copy of $\Pi B_{i}$ for each family $B_{i}$ of its objects. Assume that any 
morphism which is epic and monic is invertible.

If $F$ is a subfunctor of the restriction of $\boldsymbol{A}(R,-)$ to $\boldsymbol{B}$, then $F$ is representable by a natural equivalence $\psi: F \rightarrow \boldsymbol{B}\left(R^{\prime},-\right)$ such that the diagram

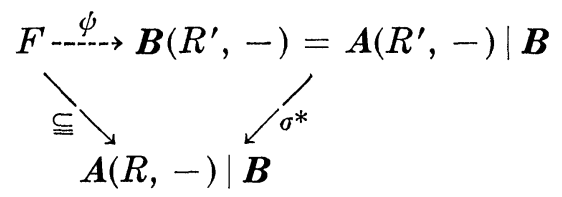

commutes for an epic $\sigma: R \rightarrow R^{\prime}$ in $A$, if and only if

(i) For each $\rho$ which is $F$ distinguished with image

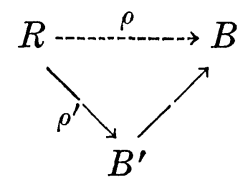

it follows that $\rho^{\prime}$ is $F$ distinguished.

(ii) $F$ is product preserving.

Proof. Let $\rho$ be $F$ distinguished with image

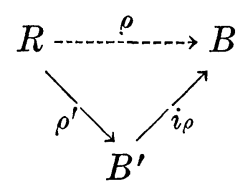

Now $\rho F$ distinguished is equivalent to the existence of a factorization

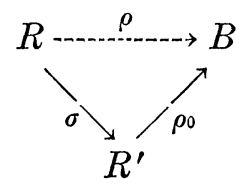

The morphism $\rho_{0}$ has an image

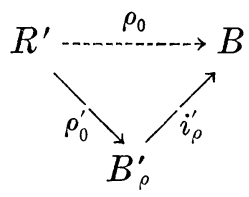

with $\rho_{0}^{\prime}$ epic in $\boldsymbol{A}$. From the minimality of $i_{\rho}$ it follows that there exists $\varphi$ such that 


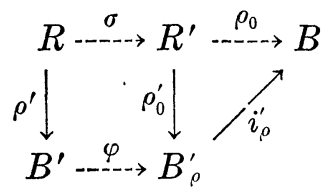

commutes. The morphisms $\sigma$ and $\rho_{0}^{\prime}$ are epic. Thus $\varphi$ is monic and epic and hence an equivalence. The object $B^{\prime} \in \boldsymbol{B}$ since $\boldsymbol{B}$ is closed under $\boldsymbol{A}$ subobjects and $\rho^{\prime}$ factors through $\sigma$. Hence $\rho^{\prime}$ is $F$ distinguished. The converse follows from the preceding theorem.

4. Applications. Let $\Omega=\cup \Omega(n)$ be a disjoint union of sets indexed by the nonnegative integers. Then $\Omega$ is called an operator set. $A$ is an $\Omega$ algebra if $A$ is a set with functions $\omega: A^{n} \rightarrow A$ defined for each $\bar{\omega} \in \Omega(n) . \quad \alpha: A \rightarrow B$ is a morphism of $\Omega$ algebras if $\alpha$ is a set mapping such that

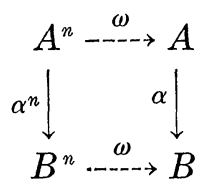

commutes for each $\omega \in \Omega(n)$ and each integer $n$. For fixed $\Omega$ let $(\Omega)$ be the category of all $\Omega$ algebras and their homomorphisms. For further details see Cohn [2].

LEmma. (a) There is only one $\theta$ algebra structure on the cartesian product of $\theta$ algebras so that each of the projections becomes a morphism of $\theta$ algebras.

(b) If $f: C_{1} \rightarrow C_{2}$ is any function, and $g: C_{2} \rightarrow C_{3}$ is a $\theta$ algebra monomorphism such that $g f$ is a $\theta$ algebra morphism, then $f$ is a $\theta$ algebra morphism.

Theorem. Let

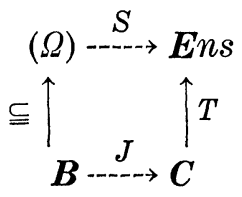

be a commutative diagram of categories where $\boldsymbol{C}$ is a full subcategory of $\theta$ algebras for some operator set $\theta, \boldsymbol{B}$ is a variety of $\Omega$ algebras, and $S, T$ are forgetful functors. Then $J$ has an adjoint.

Proof. The forgetful functor $S:(\Omega) \rightarrow \boldsymbol{E} n s$ has an adjoint $W$ by a result of Cohn [2]. Thus there is a natural equivalence 


$$
\varphi: \boldsymbol{E n s}(T C, S-) \rightarrow(\Omega)(W T C,-) \text {. }
$$

It is sufficient to show that $C(C, J-)$ is representable for each $C \in C$. A natural equivalence between $C(C, J-)$ and $F=$ a subfunctor of the restriction of $(\Omega)(W T C,-)$ to $B$ is determined by $\alpha \cdots \varphi(T \alpha)$ for $\alpha \in C(C, J B)$.

Let $\varphi(T \alpha)$ be an $F$ distinguished morphism with image

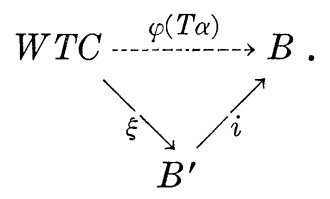

$\boldsymbol{B}$ is closed under subobjects. Thus $B^{\prime} \stackrel{i}{-\cdots} B \in \boldsymbol{B}$. Under $\varphi^{-1}$ the diagram becomes

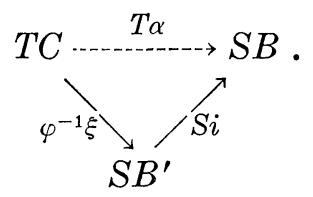

$S i=T J i$. Hence $T \alpha$ and the monomorphism $T J i$ are $\theta$ algebra homomorphisms and thus so is $\varphi^{-1} \xi$ by part (b) of the lemma. Thus $\varphi^{-1} \xi=T \alpha^{\prime}$ for some $\alpha^{\prime} \in C$ and $\xi=\varphi\left(T \alpha^{\prime}\right)$ is $F$ distinguished.

Let $\Pi B_{j} \cdots B_{j}$ be a product in $B . J\left(\Pi B_{j}\right)$ is the set theoretic cartesian product of the $\theta$ algebras $J B_{j}$ since $S=T J$ is forgetful on B. Thus $J\left(\Pi B_{j}\right)=\Pi J B_{j}$ by part (a) of the lemma. Hence $J$ and thus $F$ preserve products.

It should be noted that the same result holds by the same type of argument if there are elements of $\Omega$ corresponding to infinitary as well as finitary operations.

The preceding theorem has the following result of Lawvere [6] as a corollary.

CoRollary. Every algebraic functor has an adjoint.

Proof. An algebraic functor $\delta^{(f)}: \delta^{\left(N^{\prime}\right)} \rightarrow \delta^{(\boldsymbol{M})}$ is determined by a morphism $f: M \rightarrow N$ of algebraic theories. $U_{N}=U_{M} \delta^{(f)}$ for $U_{N}: \delta^{(\boldsymbol{N})} \cdots \rightarrow$ $\boldsymbol{E} n s$ the underlying set functor. A commutative diagram

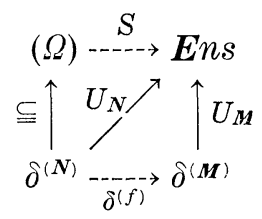


is thus obtained. This completes the proof since $\delta^{(N)}$ is a variety of $\Omega$ algebras for some operator set $\Omega$.

Let $\boldsymbol{B}$ be the category of associative $R$ algebras and let $C$ be the category of Jordan algebras over $R$. We suppose that $(\Gamma)$ is the category of all sets having the same number of $n$-ary operations defined as $\boldsymbol{B}$ for each $n \geqq 0$. If $M(C, B)$ is the set of Jordan representations $C \cdots B$, then the representability of $M(C,-): B \cdots$ Ens follows from that of $\boldsymbol{E} n s(T C, S-):\left(I^{\prime}\right) \cdots \boldsymbol{E} n s$ by the relative representability theorem for $S:(\Gamma) \cdots E$ Ens and $T: C \rightarrow E$ Ens the forgetful functors. In terms of universal algebra the representability of $M(C,-)$ is equivalent to the usual result that there exists a Jordan representation $C \cdots U C$ which is universal for any Jordan representation $C \cdots B$.

\section{REFERENCES}

1. J. Benabou, Critères de représentabilité des foncteurs, C. R. Acad. Sci. Paris 260 (1965), 1-4.

2. P. Cohn, Universal Algebra, Harper and Row, New York, 1965.

3. S. Eilenberg and S. Mac Lane, General theory of natural equivalences, Trans. Amer. Math. Soc. 58 (1945), 231-294.

4. P. Freyd, Abelian Categories, Harper and Row, New York, 1964.

5. D. M. Kan, Adjoint functors, Trans. Amer. Math. Soc. 87 (1958), 294-329.

6. F. W. Lawvere, Functorial semantics of algebraic theories, Columbia University, Dissertation, 1963; summarized in Proc. Nat. Acad. Sci. 50 (1963), 869-872.

7. S. Mac Lane, Categorical algebra, Bull. Amer. Math. Soc. 71 (1965), 40-106.

8. B. Mitchell, Theory of Categories, Academic Press, 1965.

Received March 15, 1966, and in revised form June 28, 1966. This is a summary of part of a dissertation submitted to the University of Chicago in partial fulfillment of the requirements for the degree of Doctor of Philosophy. The author is grateful to Professor Saunders Mac Lane for his helpful guidance and encouragement and to Professors P. M. Cohn and P. Freyd for the inspiration provided by their work.

UNIVERSITY OF CHICAGO AND

Goethe Universität, Frankfurt, Germany 



\section{PACIFIC JOURNAL OF MATHEMATICS}

\section{EDITORS}

\section{H. ROYDEN \\ Stanford University \\ Stanford, California}

\author{
J. P. JANS \\ University of Washington \\ Seattle, Washington 98105
}

J. DugundJI

Department of Mathematics

Rice University

Houston, Texas 77001

RichaRd ARENS

University of California

Los Angeles, California 90024

\section{ASSOCIATE EDITORS}
E. F. BECKENBACH
B. H. NeumanN
F. WOLF
K. YOSIDA

\section{SUPPORTING INSTITUTIONS}

\author{
UNIVERSITY OF BRITISH COLUMBIA \\ CALIFORNIA INSTITUTE OF TECHNOLOGY \\ UNIVERSITY OF CALIFORNIA \\ MONTANA STATE UNIVERSITY \\ UNIVERSITY OF NEVADA \\ NEW MEXICO STATE UNIVERSITY \\ OREGON STATE UNIVERSITY \\ UNIVERSITY OF OREGON \\ OSAKA UNIVERSITY \\ UNIVERSITY OF SOUTHERN CALIFORNIA
}

\author{
STANFORD UNIVERSITY \\ UNIVERSITY OF TOKYO \\ UNIVERSITY OF UTAH \\ WASHINGTON STATE UNIVERSITY \\ UNIVERSITY OF WASHINGTON

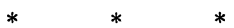 \\ AMERICAN MATHEMATICAL SOCIETY \\ CHEVRON RESEARCH CORPORATION \\ TRW SYSTEMS \\ NAVAL ORDNANCE TEST STATION
}

Mathematical papers intended for publication in the Pacific Journal of Mathematics should be typewritten (double spaced). The first paragraph or two must be capable of being used separately as a synopsis of the entire paper. It should not contain references to the bibliography. Manuscripts may be sent to any one of the four editors. All other communications to the editors should be addressed to the managing editor, Richard Arens at the University of California, Los Angeles, California 90024.

50 reprints per author of each article are furnished free of charge; additional copies may be obtained at cost in multiples of 50 .

The Pacific Journal of Mathematics is published monthly. Effective with Volume 16 the price per volume (3 numbers) is $\$ 8.00$; single issues, $\$ 3.00$. Special price for current issues to individual faculty members of supporting institutions and to individual members of the American Mathematical Society: $\$ 4.00$ per volume; single issues $\$ 1.50$. Back numbers are available.

Subscriptions, orders for back numbers, and changes of address should be sent to Pacific Journal of Mathematics, 103 Highland Boulevard, Berkeley 8, California.

Printed at Kokusai Bunken Insatsusha (International Academic Printing Co., Ltd.), 7-17, Fujimi 2-chome, Chiyoda-ku, Tokyo, Japan.

PUBLISHED BY PACIFIC JOURNAL OF MATHEMATICS, A NON-PROFIT CORPORATION

The Supporting Institutions listed above contribute to the cost of publication of this Journal, but they are not owners or publishers and have no responsibility for its content or policies. 


\section{Pacific Journal of Mathematics}

\section{Vol. 23, No. 2 \\ April, 1967}

Herbert Stanley Bear, Jr. and Bertram John Walsh, Integral kernel for

one-part function spaces .......................... 209

Mario Borelli, Some results on ampleness and divisorial schemes ....... 217

John A. Erdos, Unitary invariants for nests . ................... 229

Nathaniel Grossman, The volume of a totally-geodesic hypersurface in a pinched manifold.................................. 257

D. M. Hyman, A generalization of the Borsuk-Whitehead-Hanner theorem ............................................. 263

I. Martin (Irving) Isaacs, Finite groups with small character degrees and large prime divisors ............................. 273

I. Martin (Irving) Isaacs, Two solvability theorems ................ 281

William Lee Johnson, The characteristic function of a harmonic function in a locally Euclidean space ............................... 291

Ralph David Kopperman, Application of infinitary languages to metric spaces ............................................. 299

John Lauchlin MacDonald, Relative functor representability ............ 311

Mahendra Ganpatrao Nadkarni, A class of measures on the Bohr group.... 321

Keith Lowell Phillips, Hilbert transforms for the p-adic and p-series fields....................................... 329

Norman R. Reilly and Herman Edward Scheiblich, Congruences on regular semigroups ...

Neil William Rickert, Measures whose range is a ball ... . .

Gideon Schwarz, Variations on vector measures

Ronald Cameron Riddell, Spectral concentration for self-adjoint operators. .

Haskell Paul Rosenthal, A characterization of restrictions of

Fourier-Stieltjes transforms ................... 